\title{
Modelling and Scheduling of an Asynchronous Cyclic Production Line with Multiple Parts
}

\author{
BARIS TAN and SELCUK KARABATI \\ Graduate School of Business, Koç University \\ Çayýr Cad. No. 5 Istinye Istanbul \\ Phone: 90-212-229 3006 \\ Fax: 90-212-229 6674 \\ e-mail:btan@ku.edu.tr,skarabati@ku.edu.tr
}

June 2000

\begin{abstract}
In this study, we model and analyze a production line with asynchronous part transfers, processing time variability, and cyclic scheduling in the same framework. We consider a production line with multiple parts and finite interstation buffers. The line produces a batch of $n$ jobs repetitively using the same order of jobs in every batch. Processing time of a job on a station is a random variable and it is assumed to have a phase-type distribution. Parts are transferred between the stations in an asynchronous manner. We first present a continuous time Markov chain model to analyze the performance of this system for a given sequence. A state-space representation of the model and the associated rate matrix are generated automatically. The steady state probabilities of the Markov chain are determined by using a recursive method that exploits the special structure of the rate matrix. The cycle time, the production rate, and the expected Work-In-Progress (WIP) inventory are used as the main performance measures. We then present an approximate procedure to determine the best cyclic sequence that minimizes the cycle time. We investigate the effects of operating decisions, system structure, processing time variability, and their interaction in the same framework. Numerical results for the performance evaluation and scheduling of cyclic production lines are also presented.
\end{abstract}

Key words: Cyclic Scheduling, Stochastic Scheduling, Asynchronous Production Lines, Continuous time Markov chain models of manufacturing systems. 


\section{Modelling and Scheduling of an Asynchronous Cyclic Production Line with Multiple Parts}

\section{Introduction}

The success of a manufacturing process is largely determined by its ability to support the manufacturing task, which is formulated with the objective of meeting the requirements of the marketplace. The demand of the marketplace for more flexibility has changed manufacturing practices. The emphasis of JIT on increasing delivery speed and reliability, and reducing setup times has paved the way for the development of new design and operational policies for the management of manufacturing systems. The production line concept has been re-defined to better support the manufacturing task under increasing product variety and decreasing volumes. The simultaneous manufacturing of a mix of products has become a primary objective in the management of medium to high volume production lines. In this paper, we consider an asynchronous production line which is operated under a cyclic scheduling policy. Cyclic scheduling policies have proved to be very effective in repetitive manufacturing environments within the JIT context. ${ }^{1}$ In this approach, the minimal product set (i.e., the smallest product set having the same product mix ratio with the forecasted demand for various items), or a multiple of it, is cyclically produced. Therefore, for an effective implementation of the cyclic scheduling approach, we require that the production line be operated with zero (or negligible) setup times.

In an earlier study, we analyzed a synchronous cyclic production line. ${ }^{2}$ In this study, we extend our approach to model and analyze an asynchronous production line that produces multiple parts. More specifically, we present a performance evaluation methodology that determines the desired measures of interest when the jobs are sequenced according to a given order, and then we develop an approximate solution procedure to minimize the cycle time. We assume that there are finite interstation buffers. By following the JIT concept, the capacities of these buffers can also be set to zero. The processing time of a given job on a given station is assumed to be a random variable that follows a phase-type distribution. We consider a special case where the processing times follow a two-phase Coxian distribution separately. A state-space representation of the model and the rate matrix of the associated continuous time Markov chain are generated automatically. The steady state probabilities are then obtained by using a recursive method that exploits the special structure of the rate matrix. The proposed method yields the desired performance measures very efficiently. The cycle time, the production rate, and the expected WIP inventory are considered as the main performance measures. Sequencing decisions have profound effects on the performance. Furthermore, it is possible to improve the performance of the line by reducing the processing time variability, or alternatively, by placing interstation buffers to absorb the effects of variability. Processing time variability and buffer allocation also affect the optimal sequence that minimizes the cycle time. The model we propose allows one to investigate the effects of operating decisions, system structure, processing time variability, and also their interaction in the same framework.

The performance of repetitive manufacturing systems with deterministic processing times has also been analyzed by using Petri Nets. ${ }^{3,4}$ For the stochastic case, bounds for the production rate have been established by using stochastic timed Petri Nets. ${ }^{5}$ Rao and Jackson ${ }^{6}$ present a cyclic scheduling model of a repetitive synchronous manufacturing system with identical jobs to estimate the two-moment behavior of throughput and 
flow time of the system. They focus on the stochastic production-timing problem to find robust schedules that exhibit low variance of throughput and flow time. Bowman and Muckstadt ${ }^{7}$ consider the cyclic scheduling of operations in a multi-machine environment with infinite capacity buffers before the machines and random processing times. They analyze the performance of cyclic schedules by using a continuous time Markov chain model. Lee and $\mathrm{Seo}^{8}$ also use Markovian models to analyze stochastic cyclic flow lines with infinite interstation buffers. Zhang and Graves ${ }^{9}$ study the effects of machine failures in a cyclic production line. To the best of our knowledge, no other study in the literature investigates the cyclic scheduling problem in an asynchronous production line with finite buffers and processing time variability.

\section{General Structure}

The model we present in this study is comprised of two main blocks: performance evaluation block and scheduling block. The performance evaluation block determines the measures of interest such as the cycle time, the production rate and the average WIP inventory when a specific sequence is given. The scheduling block then suggests a new sequence to be evaluated until the desired sequence such as the one that minimizes the cycle time is obtained. Figure 1 below depicts the general structure of the model, the blocks and their interaction.

\section{Model Parameters}

(Number of stations, number of jobs, processing time parameters (mean and variance), interstation buffer capacities)

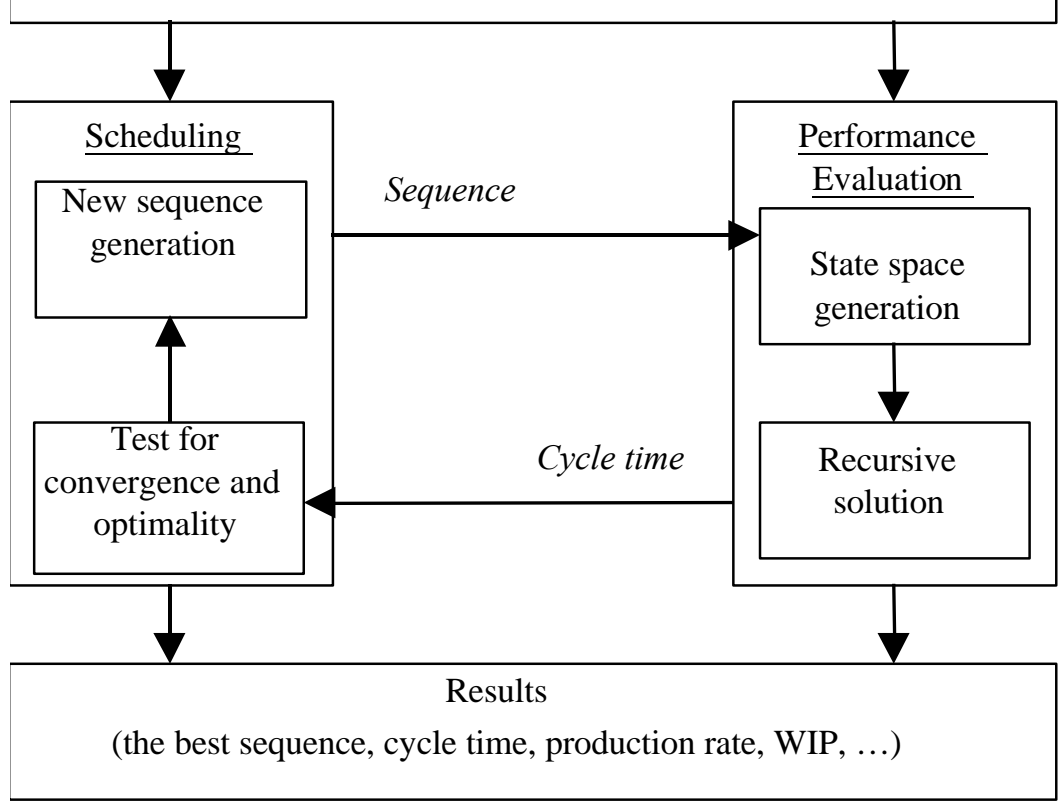

Figure 1 Performance evaluation and scheduling blocks and their interaction.

Following this dichotomy, the contribution of this study is threefold. First, we model and analyze the performance of an asynchronous production line with finite interstation buffers and multiple parts. Although, there are a number of studies on the performance evaluation of production systems in the literature, the number of studies on the performance evaluation of production lines with multiple parts is limited. ${ }^{10}$ Second, an 
approximation method for the stochastic scheduling problem is proposed. Finally, these blocks are combined in the same framework to analyze the effects of 1) operational decisions, i.e., the way the parts are sequenced, and the way the parts are transferred, 2) the structure of the production line, i.e., the number of stations and the interstation buffer capacities, and 3) processing time variability.

The remaining part of this paper is organized as follows: we first list our modeling assumptions. Then, a state space model is presented, and a recursive solution procedure for the steady-state probabilities is suggested. Subsequently, the method that generates the state space and the rate matrix automatically is discussed. Following the performance evaluation block, the scheduling block is presented. In this section, an approximate solution procedure for the cyclic scheduling problem is given. Then, numerical results that investigate the effects of operating decisions, system structure, and processing time variability are provided. Finally, our concluding remarks are given.

\section{Model Assumptions}

We consider a serial production line with $m$ stations $S T_{1}, S T_{2} \ldots S T_{m}$ and $m$-1 interstation buffers $F_{1}, F_{2}$, $\ldots, F_{m-1}$ with capacities $B_{1}, B_{2}, \ldots, B_{m-1}$. The $n$ jobs, $J_{1}, J_{2} \ldots J_{n}$, are scheduled cyclically, i.e., we repetitively produce the batch of $n$ jobs using the same order of jobs in every batch. Let $T=J_{[1]} J_{[2] \ldots J_{[n]}}$ denote a cyclic sequence of jobs, where $J_{[i]}$ is the index of the job that comes to the $i$ th position in $T$. The jobs are released to the assembly line according to the $J_{[1]} J_{[2]} \ldots J_{[\mathrm{n}]} J_{[1]} J_{[2] \ldots} J_{[\mathrm{n}]} J_{[1]} J_{[2] \ldots} J_{[\mathrm{n}]} \ldots$ sequence in a repetitive manner. Figure 2 below shows a production line with 3 stations and two finite buffers. The sample locations of 4 jobs produced according to the sequence $T=J_{[1]} J_{[2]} J_{[3]} J_{[4]}$ are shown in the figure.

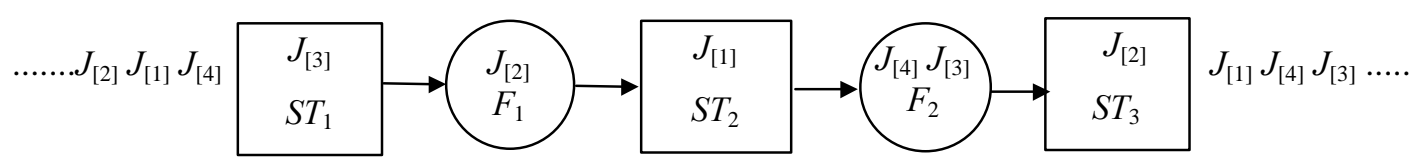

Figure 2 A production line with 3 stations and 4 jobs and sequence $J_{[1]} J_{[2]} J_{[3]} J_{[4]}$.

We assume that job transfers between the stations are done in an asynchronous manner. In an asynchronous flow line, job transfers between the stations are not coordinated, and each job, upon completion of its operation, can be transferred to its downstream station or buffer location as soon as the downstream station or buffer location becomes available. There is a continuous input stream of jobs to the first station and there is an infinite capacity shipping station after the last station. Thus the first station is never starved and the last station is never blocked.

The processing time of job $J_{i}$ on station $S T_{j}, p_{i, j}, i=1,2, \ldots, n ; j=1,2, \ldots, m$, is assumed to be a phase-type random variable with mean $1 / \mu_{i, j}$, and squared coefficient of variation $s c v_{i, j}$, and it is also independent of the other processing times. We denote the number of phases of service by $H$. For a discussion of phase-type distributions, the reader is referred to Buzacott and Shantikumar. ${ }^{12}$ 


\section{A State-Space Model}

We use a state-space model to describe this system. The state of the system at time $t$ can be described by the vector $x(t)=\left[s_{1}(t), b_{1}(t), s_{2}(t), b_{2}(t), \ldots, s_{m}(t)\right]$ where $s_{j}(t)$ is the state of station $S T_{j}$ and $b_{j}(t)$ is the number of parts in buffer $F_{j}$ at time $t$. At a given time, station $S T_{j}$ can either be working on the $h$ th phase of job $J_{i}$ ( $\mathrm{W} h h_{i}$, $h=1, . ., H ; i=1,2, \ldots, n)$, blocked (B), or idle (I). Since the first station cannot starve and the last station cannot be blocked, $s_{1}(t) \in\left\{\mathrm{W} h_{i}, \mathrm{~B}\right\}, s_{j}(t) \in\left\{\mathrm{W} h_{i}, \mathrm{~B}, \mathrm{I}\right\}, j=2,3, \ldots, m-1$, and $s_{m}(t) \in\left\{\mathrm{W} h_{i}, \mathrm{I}\right\}$, where $h=1,2, . ., H, i=1,2, \ldots$, $n$, for all cases. Since the service times at each phase are exponential random variables, the process $\{x(t): t \geq 0\}$ is a continuous time Markov chain defined on the state space $S$.

Let us assume that there is a single job type. In this case, the state of the system will only include the information about the states of the machines, i.e., whether they are working, blocked, or idle, and the information about the number of parts in each buffer. Furthermore, it will show the distribution of parts along the production line. When there are more than one job type, the state space of the single part type system is replicated as many times as the number of jobs to include the information about the locations of each job type. Note that increasing the number of job types will not affect the machine states but it will affect the locations of these jobs on the machines. Considering the position of a given job on a given station, there are $n$ different relative locations of jobs in the system. Each of these relative locations is referred as a cycle. The states in the state space are ordered in the same way for each cycle.

Then the infinitesimal generator or the rate matrix of the process $\{x(t), t \geq 0\}, \mathbf{Q}=\left\{q_{i, j}\right\}$, can be written in the following form

$$
\mathbf{Q}=\left[\begin{array}{ccccccc}
A_{1} & B_{1} & 0 & 0 & . & 0 & 0 \\
0 & A_{2} & B_{2} & 0 & . & 0 & 0 \\
0 & 0 & A_{3} & B_{3} & . & 0 & 0 \\
. & . & . & . & . & . & . \\
0 & 0 & 0 & 0 & . & B_{n-1} & 0 \\
0 & 0 & 0 & 0 & . & A_{n-1} & B_{n-1} \\
B_{n} & 0 & 0 & 0 & . & 0 & A_{n}
\end{array}\right]
$$

where $A_{i} i=1,2, \ldots, n$ is a square submatrix whose non-diagonal elements are the transition rates among the states in the same cycle, and $B_{i} i=1,2, \ldots, n$ is a square matrix whose elements are the transition rates from a state in a given cycle to a state in the following cycle. This general form holds for all phase-type distributions and for production system with finite or no interstation buffers. Note that, if there is only one job type, then $A_{1}+B_{1}$ will be the rate matrix for a production line with $m$ stations and finite buffers. This system has been studied by many researchers. ${ }^{12,13}$

In order to illustrate the cycle concept, let us consider a production line with two stations with a finite buffer and $n$ jobs. Let us assume that the processing times are exponential. The state of this system at time $t$ is $x(t)=\left[s_{1}(t), b_{1}(t), s_{2}(t)\right]$ where $s_{1}(t) \in\left\{\mathrm{W}_{i}, \mathrm{~B}\right\}$, and $s_{2} \in\left\{\mathrm{W}_{i}, \mathrm{I}\right\},\left(i=1,2, \ldots, n, b_{1}(t)=0,1, \ldots, B_{1}\right) . \quad$ In this notation, $\mathrm{W}_{i}$ is replaced with $\mathrm{W}_{i}$ for brevity, since there is only one phase. Let us first assume that there are 4 jobs and the buffer capacity is 2 . There are 20 states in the state-space. The state-transition diagram of this system is depicted in Figure 3. 


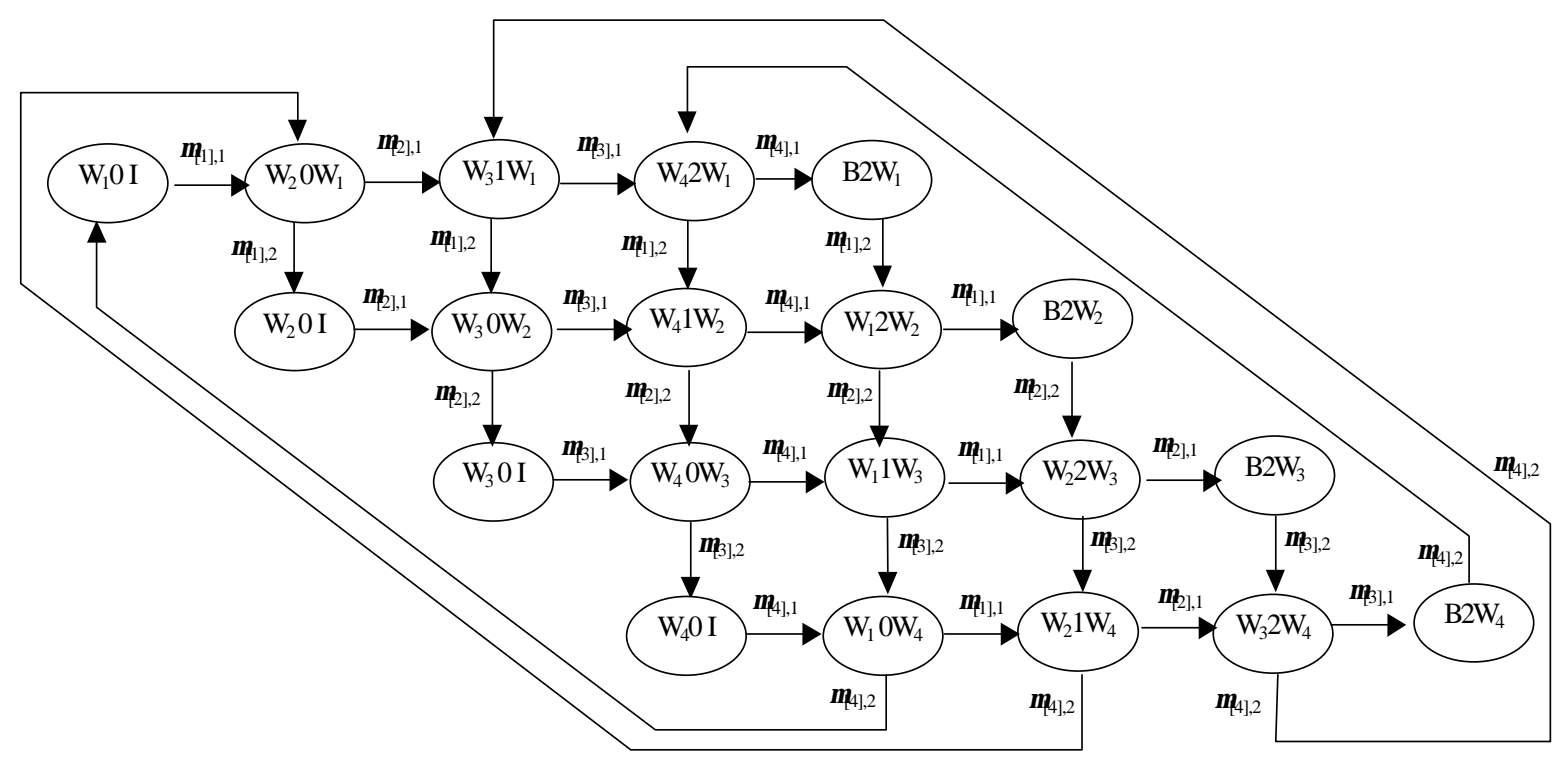

Figure 3 The state-transition diagram of a production line with 2 stations and 4 jobs, $B_{1}=2$.

We can order the states in the state space as

$$
\begin{array}{r}
S=\left\{\left[\mathrm{W}_{1}, 0, \mathrm{I}\right],\left[\mathrm{W}_{2}, 0, \mathrm{~W}_{1}\right],\left[\mathrm{W}_{3}, 1, \mathrm{~W}_{1}\right],\left[\mathrm{W}_{4}, 2, \mathrm{~W}_{1}\right],\left[\mathrm{B}, 2, \mathrm{~W}_{1}\right]\right. \\
{\left[\mathrm{W}_{2}, 0, \mathrm{I}\right],\left[\mathrm{W}_{3}, 0, \mathrm{~W}_{2}\right],\left[\mathrm{W}_{4}, 1, \mathrm{~W}_{2}\right],\left[\mathrm{W}_{1}, 2, \mathrm{~W}_{2}\right],\left[\mathrm{B}, 2, \mathrm{~W}_{2}\right]} \\
{\left[\mathrm{W}_{3}, 0, \mathrm{I}\right],\left[\mathrm{W}_{4}, 0, \mathrm{~W}_{3}\right],\left[\mathrm{W}_{1}, 1, \mathrm{~W}_{3}\right],\left[\mathrm{W}_{2}, 2, \mathrm{~W}_{3}\right],\left[\mathrm{B}, 2, \mathrm{~W}_{3}\right]} \\
\left.\left[\mathrm{W}_{4}, 0, \mathrm{I}\right],\left[\mathrm{W}_{1}, 0, \mathrm{~W}_{4}\right],\left[\mathrm{W}_{2}, 1, \mathrm{~W}_{4}\right],\left[\mathrm{W}_{3}, 2, \mathrm{~W}_{4}\right],\left[\mathrm{B}, 2, \mathrm{~W}_{4}\right]\right\}
\end{array}
$$

According to this ordering, there are four cycles with 5 states in each cycle. If there are $n$ different jobs, there will be $n$ cycles and each cycle will include 5 states, given for cycle $i$ as

$$
\left[\mathrm{W}_{[i]}, 0, \mathrm{I}\right],\left[\mathrm{W}_{[i+1 \bmod n]}, 0, \mathrm{~W}_{[i]}\right],\left[\mathrm{W}_{[i+2 \bmod n]}, 1, \mathrm{~W}_{[i]}\right],\left[\mathrm{W}_{[i+3 \bmod n]}, 2, \mathrm{~W}_{[i]}\right],\left[\mathrm{B}, 2, \mathrm{~W}_{[i]}\right]
$$

where mod denotes the modulus function with the convention that $(n \bmod n)=n$. That is, $[i \bmod n]$ is the remainder after division of $i$ to $n$ when $i>n, i$ when $0<i \leq n$, and $n$ when $i=0$.

Determining the submatrices $A_{i}$ and $B_{i}$ for cycle $i, i=1,2, \ldots, n$, completely describes the rate matrix $\mathbf{Q}$. For this specific example, the submatrices $A_{i}$ and $B_{i}$ for cycle $i$ can be written as

$$
\begin{aligned}
& A_{i}=\left[\begin{array}{ccccc}
-\mu_{[i], 1} & \mu_{[i], 1} & 0 & 0 & 0 \\
0 & -\mu_{[i+1, \bmod n], 1}-\mu_{[i], 2} & \mu_{[i+1, \bmod n], 1} & 0 & 0 \\
0 & 0 & -\mu_{[i+2, \bmod n], 1}-\mu_{[i], 2} & \mu_{[i+2], 1} & 0 \\
0 & 0 & 0 & -\mu_{[i+3, \bmod n], 1}-\mu_{[i], 1} & \mu_{[i+3, \bmod n], 1} \\
0 & 0 & 0 & 0 & -\mu_{[i], 2}
\end{array}\right] \\
& B_{i}=\left[\begin{array}{ccccc}
0 & 0 & 0 & 0 & 0 \\
\mu_{[i], 2} & 0 & 0 & 0 & 0 \\
0 & \mu_{[i], 2} & 0 & 0 & 0 \\
0 & 0 & \mu_{[i], 2} & 0 & 0 \\
0 & 0 & 0 & \mu_{[i], 2} & 0
\end{array}\right]
\end{aligned}
$$


Let $\boldsymbol{P}(t)$ be the row vector of state probabilities with its $i$ th component denoting the probability that the state of the system is $i$ at time $t$, i.e., $P_{i}(\mathrm{t})=P[X(t)=i]$. Given the initial state probability vector $\boldsymbol{P}(0), \boldsymbol{P}(\mathrm{t})$ satisfies the Kolmogorov differential equations:

$$
\frac{d}{d t} \boldsymbol{P}(t)=\boldsymbol{P}(t) \mathbf{Q}
$$

The rate matrix given in Equation (1) shows that there exists a single communicating class in the process $\{X(t): t \geq 0\}$, and the process is ergodic. Therefore, the steady-state probabilities exist. Let $\pi$ be the row vector of steady-state probabilities with its $i$ th component denoting the steady-state probability that the state of the system is $i$ at time $t$, i.e., $\pi_{i}=\lim _{t \rightarrow \infty} P_{i}(\mathrm{t})$. The steady-state probability vector can be determined from the following equations:

$$
\begin{aligned}
& \pi \mathbf{Q}=0, \\
& \pi \underline{u}=1 .
\end{aligned}
$$

where $\underline{u}$ is a column vector of ones.

Once the steady-state probabilities are available, a number of performance measures can be determined. One of the most important performance measures is the production rate or the throughput. In this study, we define the production rate, $p r$, as the number of jobs of a particular type produced per unit time in the long run. Alternatively, it can be defined as the total number of jobs produced per unit time. If there are $n$ different jobs in a batch, the production rate defined based on the number of jobs of a particular type is $1 / n$ of the production rate defined based on the total number of jobs produced. For example, if a sequence of jobs A, B, C is produced cyclically with a production rate of one job of type A per minute (equivalently one B and one C per minute), the total number of parts produced per minute will be three jobs per minute.

The expected time between completion times of successive batches, i.e., the time between completion times of jobs of a particular type is also of interest. This inter-departure time is called the cycle time, CT. The cycle time is the reciprocal of the production rate is determined based on the jobs of a particular type, i.e., CT= $1 / p r$.

Since there is no scrapping of materials, the rate at which the products are processed is the same for all the stations as a result of the conservation of materials. Thus the production rate of the production line, $p r$, can be determined by multiplying the probability that a given station is processing a given phase of a job with its job completion rate $r_{k}$ at that state, i.e.,

$$
p r=\sum_{k \in Z([i], j, h)} \pi_{k} r_{k}, \quad i=1,2, \ldots, n \text { and } j=1,2, \ldots, m
$$

where $Z\left([i]_{j}, h\right)$ is the set of the states where station $S T_{j}$ is processing the $h$ th phase of job $J_{i}, h=1, \ldots, H$. Note that, $r_{k}$ can be computed from the transition rates of a given phase-type distribution. When $H=1$, that is when the processing times are exponentially distributed we have

$$
p r=\mu_{[i], j} \sum_{k \in Z([i], j, 1)} \pi_{k} \quad i=1,2, \ldots, n \text { and } j=1,2, \ldots, m
$$


Another important performance measure is the expected WIP inventory. When we consider WIP inventory, we determine both the expected number of parts and also the expected number of batches held in the production line.

Let $n_{k}$ be the total number of parts being held in the stations when the state of the system is $k$. The number of parts being held in the production line at a given state is the sum of the parts in the buffers and the parts held at the stations. Note that a station holds a part when it is working or when it is blocked.

Then the expected WIP inventory of parts, $E_{w i p}^{p}$, can easily be obtained as

$$
E_{w i p}^{p}=\sum_{k \in S} n_{k} \pi_{k}
$$

Instead of using the number of parts, one can use the expected number of batches, $E_{\text {wip }}^{b}$ as the primary WIP performance measure. In this case, $n_{k}$ is simply defined as the number of batches being held in the stations when the state of the system is $k$.

We can now determine the production rate and the expected number of parts for the above example of a two-station production line with four jobs. In order to determine the production rate, let us consider the second job being processed at the first station. The set of states where the second job is being processed at the first station is $Z(2,1,1)=\left\{\left[\mathrm{W}_{2}, 0, \mathrm{~W}_{1}\right],\left[\mathrm{W}_{2}, 0, \mathrm{I}\right],\left[\mathrm{W}_{2}, 2, \mathrm{~W}_{3}\right],\left[\mathrm{W}_{2}, 1, \mathrm{~W}_{4}\right]\right\}$. Thus the production rate is

$$
p r=\mu_{[2], 1}\left(\pi_{2}+\pi_{6}+\pi_{14}+\pi_{18}\right)
$$

and $C T=1 / p r$. Note that there are $1,2,3,4$, and 4 parts in the states $\left[\mathrm{W}_{[i]}, 0, \mathrm{I}\right],\left[\mathrm{W}_{[i+1 \bmod n]}, 0, \mathrm{~W}_{[i]}\right],\left[\mathrm{W}_{[i+2 \bmod n]}\right.$, $\left.1, \mathrm{~W}_{[i]}\right], \quad\left[\mathrm{W}_{[i+3 \bmod n]}, 2, \mathrm{~W}_{[i]}\right],\left[\mathrm{B}, 2, \mathrm{~W}_{[i]}\right]$, respectively, for cycle $i, i=1,2,3,4$. Since the states of the system are ordered in the same way for each cycle, the expected WIP inventory is

$$
E_{W I P}=\sum_{i=1}^{4} \pi_{5(i-1)+1}+2 \pi_{5(i-1)+2}+3 \pi_{5(i-1)+3}+4 \pi_{5(i-1)+4}+4 \pi_{5(i-1)+5}
$$

Although, it is easy to determine the performance measures for this small size example, both generating the state space and then determining the steady state probabilities for a large size model are quite complicated. The size of the matrix $\mathbf{Q}$ is defined by the number of elements in the state space. The number of states in the state-space increases exponentially with the number of stations in the line, polynomially with the number of phases and with the buffer capacities, and linearly with the number of jobs.

For a production line with 10 stations, 20 jobs, 3 phases, and no interstation buffers, there are 8,347,600 states in the state space. Generating this state space manually is not possible. Furthermore, solving Equations (5) and (6) directly is not computationally efficient. In the next section, we present a recursive solution procedure to determine the steady-state probabilities more efficiently. We then explain how the state space and the rate matrix are generated automatically. 


\section{A Recursive Solution Procedure for the Steady-State Probabilities}

The special structure of the rate matrix can be exploited to determine the steady-state probabilities efficiently. When there is a single part type, then the associated rate matrix has a block tridiagonal form and it can be analyzed by using matrix-geometric methods. ${ }^{14}$ However, since there are multiple parts, the submatrices $A_{i}$ and $B_{i}$ are distinct for each $i$. In this case, the special structure of the rate matrix allows us to determine the steady-state probabilities recursively. In an earlier study, we used a similar methodology to analyze an asynchronous line with exponential stations and no interstation buffers. ${ }^{16}$

Let the steady-state probability vector $\pi$ be partitioned according to the partitioning of $\mathbf{Q}$. Then Equation (5) yields

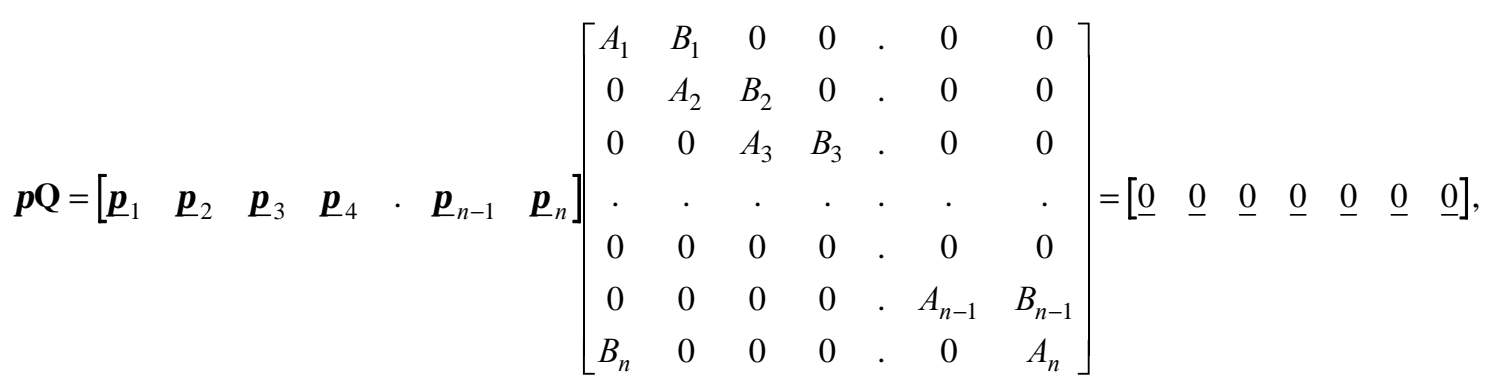

where $\underline{\pi}_{i}$ is a row vector of the same size as the sizes of submatrices $A_{i}$ and $B_{i}$, and $\underline{0}$ is a vector of zeros of the same size as $\underline{\pi}_{i}$. Direct matrix multiplication in Equation (12) yields the following system of recursive equations:

$$
\begin{aligned}
& \underline{\pi}_{1} A_{1}+\underline{\pi}_{n} B_{n}=\underline{0}, \\
& \underline{\pi}_{i} B_{i}+\underline{\pi}_{i+1} A_{i+1}=\underline{0} \quad i=1,2, \ldots, n-1 .
\end{aligned}
$$

The model generation algorithm that will be described in the following section always yields upper triangular $A_{i}$ s. Furthermore, the diagonal elements of $A_{i}$ are non-zero. Then $A_{i}$ is non-singular and $A_{i}^{-1}$ exists. In this case, Equation (14) can be rewritten as

$$
\underline{\pi}_{i+1}=-\underline{\pi}_{i} B_{i} A_{i+1}^{-1}, \quad i=1,2, \ldots, n-1,
$$

Equation (15) allows us to determine $\pi$ either directly or by using an iterative method. In order to determine $\pi$ directly, Equation (15) can be written in terms of $\underline{\pi}_{1}$ as

$$
\underline{\pi}_{i}=(-1)^{i+1} \underline{\pi}_{1} \prod_{j=1}^{i-1} B_{j} A_{j+1}^{-1} \quad i=2, \ldots, n .
$$

Inserting Equation (16) into Equation (13) for $i=n$ yields the following equation to be solved for $\pi_{1}$ :

$$
\underline{\pi}_{1}\left[A_{1}+(-1)^{n+1}\left(\prod_{j=1}^{n-1} B_{j} A_{j+1}^{-1}\right) B_{n}\right]=0 .
$$


There are infinitely many solutions of the equation given above. By using any one of these solutions in Equation (15) yields $\underline{\pi}_{i}, i=2,3, . ., n$. Normalizing the resulting vector yields the steady-state probabilities.

Alternatively, the properties of Markov chains can be exploited to determine $\pi$ by using an iterative method. $\{x(t): t \geq 0\}$ is a finite, aperiodic, and irreducible Markov chain. Therefore, the vectors $\boldsymbol{P}(t)$ converge to the stationary probability vector $\pi$, regardless of the choice of the initial vector, i.e., $\pi=\lim _{t \rightarrow \infty} \boldsymbol{P}(\mathrm{t})$.

Let $\underline{\pi}_{1}$ be initialized to an arbitrary vector. Starting with $\underline{\pi}_{1}$, new values for $\underline{\pi}_{i} i=2,3, . ., n$ can be determined recursively by using Equation (15). Once $\underline{\pi}_{n}$ is obtained, Equation (13) yields the new value for $\underline{\pi}_{1}$, i.e., $\underline{\pi}_{1}=-\underline{\pi}_{n} B_{n} A_{1}^{-1}$. Starting with this new value of $\underline{\pi}_{1}$, the same procedure can be repeated until no major changes are observed between the new and current values. Similar to the direct method, normalizing the resulting vector yields the steady-state probabilities. For a thorough discussion of iterative methods and other numerical techniques to solve Markov chains, the reader is referred to Stewart. ${ }^{17}$

The solution procedure proposed in this section is more efficient than direct determination of the steadystate probabilities from the rate matrix $\mathbf{Q}$. Furthermore since the structure of the submatrices $A_{i}$ and $B_{i}$ are the same for all $i$, they can easily be generated automatically for a given job $J_{i}$. Therefore it is not necessary to form and to store the matrix $\mathbf{Q}$ in the memory. Rapid growth of the states in the submatrices $A_{i}$ and $B_{i}$ still limits the applicability of this model to large problems. Note that, this is a general limitation of the state-space models, and it is not specific to the procedure given in this study.

\section{Generating the State Space and the Rate Matrix}

The state-space based methods are based on utilizing characteristic matrix of a given system and then analyzing this matrix for the performance measures of interest. However, it is extremely time consuming to generate the state-space and then to build the associated matrix manually. Papadopoulos and $\mathrm{O}^{\prime}$ Kelly ${ }^{18}$ generate the transition matrix of a multistation production line recursively by exploiting the nested block tridiagonal structure of the underlying quasi-birth and -death process. Namely, the repeating structure of the submatrices is used and the probability matrix is formed by using the submatrices and the state space that are given explicitly.

In our approach, we build both the state space and the rate matrix automatically. In other words, our method does not require any information regarding the submatrices and the state space, and it generates both of them as it iterates.

We start state-space generation at an arbitrary state, say, at the state where the stations are working on the first phase of a given job according to the given sequence, and the buffers are empty. We include this state in the state space list as the first state of the state space and assign an index of one. Then we consider all possible changes in the machine states. That is, for each machine that is not blocked or idle, a possible transition is either to another phase of the service, or to a service completion. For each transition, we update the relative location of the jobs along the production line and also we update the machine states that are affected by this transition. According to the locations of jobs, the machine states, and the number of parts in the buffers, we determine the state that will be visited next as a result of this transition. If this new state is not included in the state space then it is included and assigned a new index. Then, we store the index of the current state, the index of the next state, and the transition rate. This representation allows us to store a sparse matrix efficiently. 
Let us explain this method by an example of a three station exponential production line with two interstation buffers of capacity 2 , which produces 4 jobs cyclically with the sequence $J_{[1]}, J_{[2],} J_{[3]}, J_{[4]}$, Let the current state be $\left[B, 2, W_{3}, 1, W_{1}\right]$ and its index be $I_{\text {current }}$. In this state there are two possible changes: either machine 2 finishes its operation or machine 3 finishes its operation. For the first case, when the second station finishes its operation, the state of the system changes to $\left[\mathrm{W}_{1}, 2, \mathrm{~W}_{4}, 2, \mathrm{~W}_{1}\right]$ and the transition rate is the

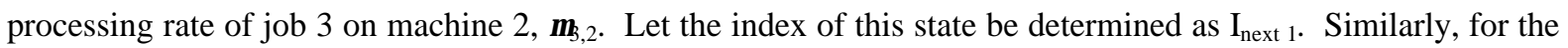
second case, when the first station finishes its operation the state of the system changes to $\left[\mathrm{B}, 2, \mathrm{~W}_{3}, 0, \mathrm{~W}_{2}\right]$ and the rate of this transition is the processing rate of job 1 on machine $3, \mu_{1,3}$. Let the index of this state be

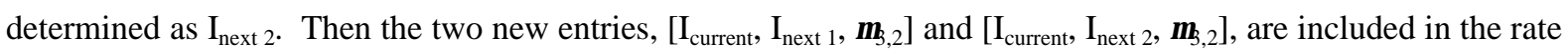
matrix list as a result of iterating the state $\left[\mathrm{B}, 2, \mathrm{~W}_{3}, 1, \mathrm{~W}_{1}\right]$.

We continue this procedure with the next state in the state space until all the states in the state space are considered. As a result, the state space and the rate matrix are built. Finally, in order to determine the production rate, we form a reward function according to a given machine and a given job. For example, if we consider the second job processed at the first station, we choose the states where the first station is up and not blocked. Denoting the chosen states with one and the others with zero yields the desired reward function.

\section{The Scheduling Problem}

The performance evaluation framework of the previous section has been developed for a fixed sequence of the parts. Note that the total number of sequences is $(n-1)$ ! As the number of jobs increases, the best sequence that maximizes (minimizes) the production rate (the cycle time) of the line cannot be determined via total enumeration. Moreover, as a special case of the stochastic model, the deterministic case has been shown to be NP-hard. ${ }^{15}$ Therefore, it is of interest to develop approximate solution procedures for the scheduling problem by using the performance evaluation block described above.

\section{Approximate Solution Procedures for the Scheduling Problem}

The approximate solution procedure is based on Equation (7), where the production rate of the line is expressed in terms of the service rate of a given operation and the probability of being in the set of states of the system where this particular operation is processed. To simplify the discussion, and without any loss of generality, we will assume that the processing times are exponential, i.e., $H=1$.

Let $T=J_{[1]} J_{[2] \ldots} J_{[n]}$ be a sequence of jobs and $\rho_{[i], j}$ be the sum of the probabilities of the states where station $S T_{j}$ is processing job $J_{[i]}$, i.e., $\rho_{[i], j}=\sum_{k \in Z_{[i], j, 1}} \pi_{k}$. The performance evaluation is performed for a fixed sequence of jobs, therefore $\rho_{[i], j}$ 's are valid for the sequence under consideration. For sequence generation purposes, however, we can assume that $\rho_{[i], j}$ 's will be valid for all sequences, and then try to re-sequence the jobs to maximize the production rate of the line with respect to current $\rho_{[i], j}$ values. Once the new sequence is determined, we can update the $\rho_{[i], j}$ values to compute the actual production rate of the new sequence, and proceed in an iterative manner with the updated $\rho_{[i], j}$ values until a stopping criterion is met. 
In order to find a new sequence for a given set of $\rho_{[i], j}$ values the following linear bottleneck assignment problem is solved:

$$
\begin{aligned}
\text { BAP: } & \max \quad \min _{i, k} c_{i, k} x_{i, k} \\
\text { st. } & \sum_{i=1}^{n} x_{i, k}=1 \quad k=1,2, \ldots, n, \\
& \sum_{k=1}^{n} x_{i, k}=1 \quad i=1,2, \ldots, n, \\
& x_{i, k} \in\{0,1\}
\end{aligned}
$$

where $c_{i, k}$ is the production rate when job $J_{i}$ is assigned to position $k$ in the new sequence under the initial assumption that $\rho_{[i], j}$ values will be valid for all sequences. When job $J_{i}$ is assigned to position $k$, the production rate can be determined in $m$ different ways, i.e., by multiplying $\rho_{[k], j}$ by $\mu_{i, j}, j=1,2, \ldots, \mathrm{m}$. Note that when job $J_{[k]}$ is assigned to position $k$ all these multiplications are equal to production rate of sequence $T=J_{[1]} J_{[2] \ldots J_{[n]}}$, because $\rho_{[i], j}$ 's are generated using sequence $T$. The objective of the sequencing problem is the maximization of the production rate, therefore $c_{i, k}$ can be considered as an approximation of the production rate, and be taken as $c_{i, k}=\min _{j} \mu_{i, j} \rho_{[k], j}$ or $c_{i, k}=\frac{1}{m} \sum_{j=1}^{m} \mu_{i, j} \rho_{[k], j}$. Therefore, the linear bottleneck assignment problem, which can be solved in polynomial time, tries to find a new sequence of jobs that maximizes the approximate production rate value. In order to prevent cycling, on the other hand, $B A P$ is solved $n$ times, and in the ith iteration the assignment problem is modified so that job $J_{[i]}$ is not assigned to position $i$ in the sequence. The approximate solution procedure can be formally stated as follows:

Step 0: Let $T=J_{[1]} J_{[2]} \ldots J_{[n]}$ be an initial sequence of the jobs. Let $G L B=0$ be a lower bound on the optimal production rate.

Step 1: Determine the production rate of sequence $T$ by finding the $\sum_{k \in Z_{[i], j, 1}} \pi_{k}$ values. Let $\rho_{[i], j}=\sum_{k \in Z_{[i], j, 1}} \pi_{k}$

Step 2: Determine the $C=\left[c_{i, k}\right]$ matrix where $c_{i, k}=\min _{j} \mu_{i, j} \rho_{[k], j}$ or $c_{i, k}=\frac{1}{m} \sum_{j=1}^{m} \mu_{i, j} \rho_{[k], j}$.

Step 3: $\quad$ Let $L B=0$. For $l=1$ to $n$

Let $C^{\prime}=C$ and $c^{\prime}{ }_{[i], i}=0$. Solve $B A P$ with $C^{\prime}$ and let $T^{\prime}=J_{[1]}, J_{[2]}, \ldots J_{[n]}$, be the resulting sequence. Determine the production rate of sequence $T^{\prime}, \operatorname{pr}\left(T^{\prime}\right)$. If $\operatorname{pr}\left(T^{\prime}\right)>G L B$, let $G L B=$ $\operatorname{pr}\left(T^{\prime}\right)$, and $T_{H}=T^{\prime}$, and if $\operatorname{pr}\left(T^{\prime}\right)>L B$, let $L B=\operatorname{pr}\left(T^{\prime}\right)$, and $T_{L O C A L B E S T}=T^{\prime}$.

Step 4: If the stopping criterion is met, STOP, $T_{H}$ is the resulting sequence; otherwise let $T=T_{\text {LOCALBEST, }}$ and go to Step 1 .

The stopping criterion of Step 4 can be the number of times Steps 1 through 4 is repeated, a target production rate, or a lower bound on the incremental change the production rate attains in successive repetitions of Step 4 . 
We now present a 3-station 3-job problem to illustrate the main iteration of the approximate solution procedure. Let $H=1$, and service rates be as follows: $\mu_{1,1}=0.2, \mu_{1,2}=0.25, \mu_{1,3}=0.5, \mu_{2,1}=0.11, \mu_{2,2}=0.11$, $\mu_{2,3}=0.33, \mu_{3,1}=1.0, \mu_{3,2}=1.0$, and $\mu_{3,3}=0.5$. Also let the initial sequence of Step 0 be $T=J_{1} J_{2} J_{3}$. The production rate of sequence $T$ is computed as 0.0388 by following the methodology presented in the preceding sections. Then $\rho_{[i], j}$ values of Step 1 are computed as follows: $\rho_{1,1}=0.1940, \rho_{1,2}=0.1552, \rho_{1,3}=0.0776, \rho_{2,1}=0.3494$, $\rho_{2,2}=0.3494, \rho_{2,3}=0.1164, \rho_{3,1}=0.0388, \rho_{3,2}=0.0338$, and $\rho_{3,3}=0.0776$. Let us assume that $c_{i, k}=\min _{j} \mu_{i, j} \rho_{[k], j}$. For example, $c_{1,2}$, i.e., the cost of assigning $J_{1}$ to the second position is computed as follows:

$$
c_{1,2}=\min \{0.20(0.3494), 0.25(0.3494), 0.5(0.1164)\}=\min \{0.0698,0.0698,0.0582\}=0.0582
$$

The resulting $C=\left[c_{i, \mathrm{j}}\right]$ matrix of Step 2 is as follows:

$$
C=\left[\begin{array}{lll}
0.0388 & 0.0582 & 0.0077 \\
0.0172 & 0.0388 & 0.0043 \\
0.0388 & 0.0582 & 0.0388
\end{array}\right]
$$

In Step 3, for $l=1$, we let $C^{\prime}=C$ and $c^{\prime}{ }_{1,1}=0$, and solve the $B A P$ with $C$ '. The solution of the bottleneck assignment problem generates sequence $T^{\prime}=J_{2} J_{1} J_{3}$. The production rate of sequence $T$ ' is computed as 0.0394 .

\section{Numerical Results}

In this section we present our computational experience with the performance evaluation and approximate solution procedures presented in the preceding sections. For the implementation of the performance evaluation procedure, a program that generates the submatrices $A_{i}$ and $B_{i}$ for a given number of stations and for a given number phases of service is developed. The submatrix $A_{i}$ generated by this program is always in upper triangular structure. Thus the inverse of this matrix, which is necessary in Equations (15) and (16), can easily be obtained with $\mathrm{o}\left(n^{2}\right)$ operations. Furthermore, by exploiting the special structure of the submatrix $B_{i}$, the matrix multiplication in these equations can be performed very efficiently. Once these submatrices are available, steady-state probabilities are obtained by using the recursive methodology presented in this study.

We first investigate the computational properties of the performance evaluation method. Figure 4 depicts the total number of floating point operations (flops) and the CPU time (in seconds) required to evaluate the performance of the models with different number of jobs and machines on a Sun Ultra II computer.

In Table 2 we report our computational experience with the approximate solution procedure. We consider 8-job and 4- and 6-station problems with exponential processing times, and determine the optimal sequences through complete enumeration. For each (number of jobs, number of stations) combination, we consider a set of 100 problems. Expected processing times are drawn from a continuous uniform $(1,100)$ distribution. For each version of the approximate solution procedure, we report the relative deviations of the obtained cycle times from optimal cycle time values as a percentage of the optimal cycle time values. 

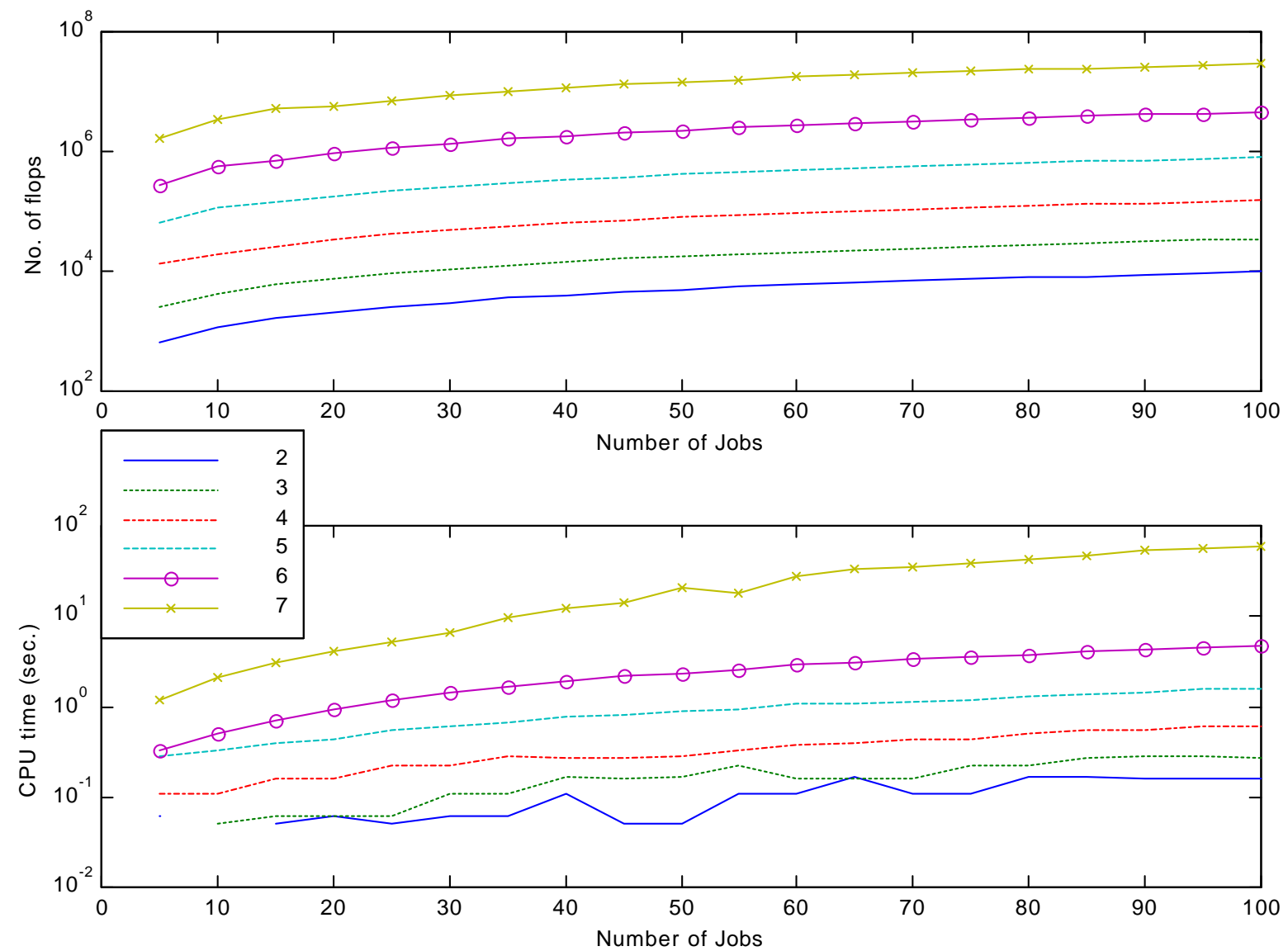

Figure 4 Computational performance of the performance evaluation block for different number of jobs and machines.

Table 2 Computational results for the approximate solution procedure.

\begin{tabular}{|c|c|c|c|c|c|}
\hline \multirow{2}{*}{$\begin{array}{c}\text { No of } \\
\text { Jobs }\end{array}$} & $\begin{array}{l}\text { No of } \\
\text { Machines }\end{array}$ & Heuristic & \multicolumn{3}{|c|}{ \% Deviation from the Optimal Value } \\
\cline { 3 - 6 } & & $c_{i, k}=\min _{j} \mu_{i, j} \rho_{[k], j}$ & 0.18 & 1.42 & 4.38 \\
\hline \multirow{2}{*}{8} & 4 & $c_{i, k}=\frac{1}{m} \sum_{j=1}^{m} \mu_{i, j} \rho_{[k}$ & 0.00 & 1.45 & 3.22 \\
\hline \multirow{2}{*}{8} & \multirow{2}{*}{6} & $c_{i, k}=\min _{j} \mu_{i, j} \rho_{[k], j}$ & 0.00 & 1.86 & 4.36 \\
\cline { 5 - 7 } & & $c_{i, k}=\frac{1}{m} \sum_{j=1}^{m} \mu_{i, j} \rho_{[k}$ & 0.00 & 1.36 & 4.61 \\
\hline
\end{tabular}

The results presented in Table 2 are obtained by starting the approximate solution procedure with a randomly chosen sequence, and iterating Steps 1 through 4 ten times. The total CPU time for a single problem has turned out to be at most $40 \mathrm{CPU}$ seconds. The results of Table 2 indicate that reasonably good sequences can be obtained with acceptable levels of computational effort. 
Although the approximate solution can be easily used for larger size problems, the lack of optimal values (or good lower bounds) hinders us from reporting the relative performance of the procedure for large size problems. In Table 3 we report our computational experience in a restricted case of the problem. We consider 2station problems for which lower bounds can be obtained by solving the deterministic counterparts of the problems. Matsuo ${ }^{20}$ has shown that 2-station deterministic asynchronous cyclic scheduling problem with no buffers between the two stations can be formulated as a Gilmore-Gomory ${ }^{21}$ TSP. For each $s c v$ value and number of jobs combination we consider 100 problems with mean processing times drawn from a continuous uniform $(1,100)$ distribution. We then report the average deviations from the lower bounds that are obtained by solving the deterministic counterparts of the problems. As the results of Table 3 demonstrate, the average deviation values deteriorate with increasing $s c v$ values. This deterioration can be mostly attributed to deterministic lower bound's poor performance when $s c v$ values are higher.

Table 3 2-Station Problems: Average \% deviations from deterministic lower bounds.

\begin{tabular}{|c|l|c|c|c|c|c|c|}
\hline \multirow{2}{*}{$\begin{array}{c}\text { No of } \\
\text { Jobs }\end{array}$} & Heuristic & \multicolumn{5}{|c|}{ scv } \\
\cline { 3 - 8 } & $c_{i, k}=\min _{j} \mu_{i, j} \rho_{[k], j}$ & 0.5 & 0.6 & 0.7 & 0.8 & 0.9 & 1.0 \\
\hline \multirow{2}{*}{15} & 19.82 & 23.72 & 30.11 & 33.49 & 35.57 & 38.83 \\
\cline { 2 - 8 } 20 & $c_{i, k}=\frac{1}{m} \sum_{j=1}^{m} \mu_{i, j} \rho_{[k}$ & 19.28 & 24.02 & 30.36 & 33.23 & 36.04 & 38.74 \\
\cline { 2 - 8 } & $c_{i, k}=\min _{j} \mu_{i, j} \rho_{[k], j}$ & 22.04 & 25.45 & 30.22 & 34.28 & 38.06 & 41.14 \\
\hline
\end{tabular}

Additional numerical experiments are conducted to investigate the effects of sequencing decisions, buffer allocation, service time variability, and transfer mode selection (asynchronous part transfer versus synchronous part transfer) on the production rate and expected WIP inventory performance measures. Since variability affects the optimal sequence that minimizes the cycle time, the interaction of variability and sequencing decisions is also investigated.

Figure 5 shows the effect of buffer capacities on the optimal cycle time of a production line with three stations, four jobs, and two interstation buffers with capacities $B_{1}$ and $B_{2}$. Processing time of each job on each machine is exponential and their means are given in $4 \times 3$ matrix $[[1,3,2] ;[2,5,6] ;[5,1,3] ;[3,4,5]]$. The figure depicts the iso-cycle time contours for different values of $B_{1}$ and $B_{2}$. For each allocation of $B_{1}$ and $B_{2}$, the optimal cycle time is determined by finding the best sequence that minimizes the cycle time. Since there are only four jobs, the optimal sequence is obtained by enumerating (4-1)!=6 different cases. The best sequence for each allocation is shown in Figure 6. Figure 5 suggests that the optimal buffer allocation that minimizes the cycle time for a given total buffer capacity can also be determined efficiently by exploiting the special structure of the contour lines. Figure 7 shows the minimum cycle time that can be obtained by allocating the total buffer capacity and determining the cyclic schedule, the optimal buffer allocation, and the optimal sequence for the same production line. 


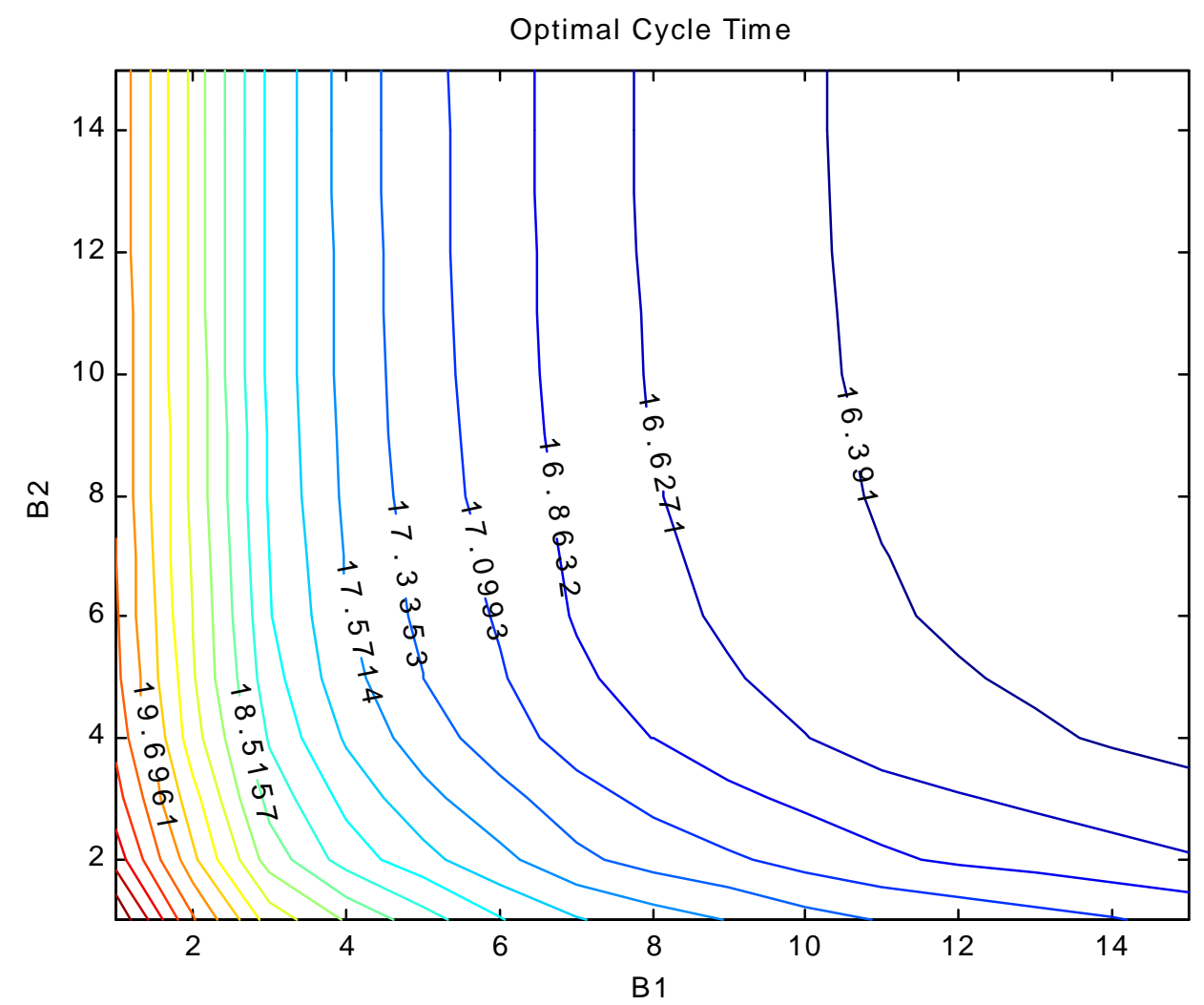

Figure 5 The effect of buffer capacities on the optimal cycle time.

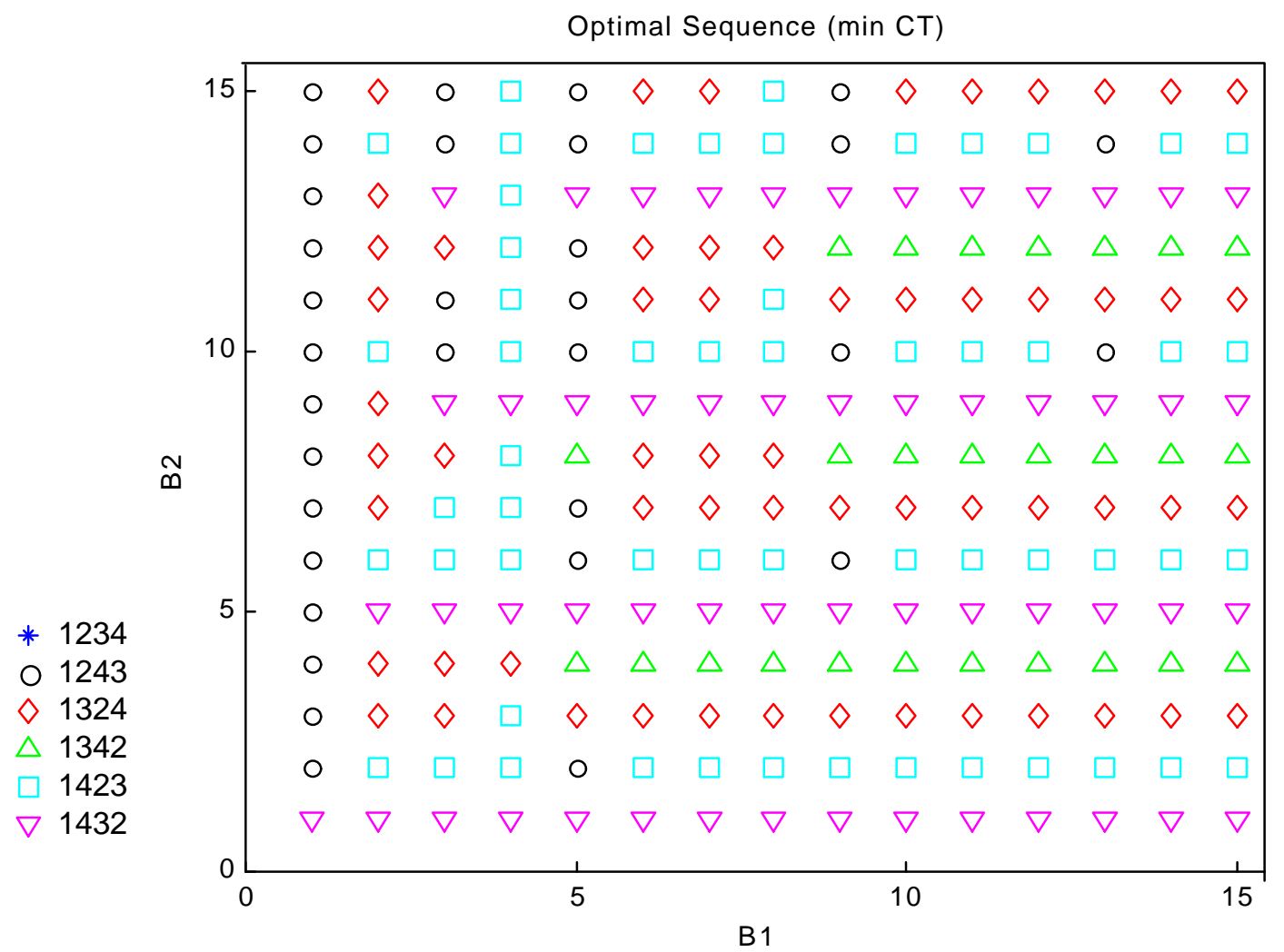

Figure 6 The effect of buffer capacities on the optimal sequence that minimizes the cycle time. 


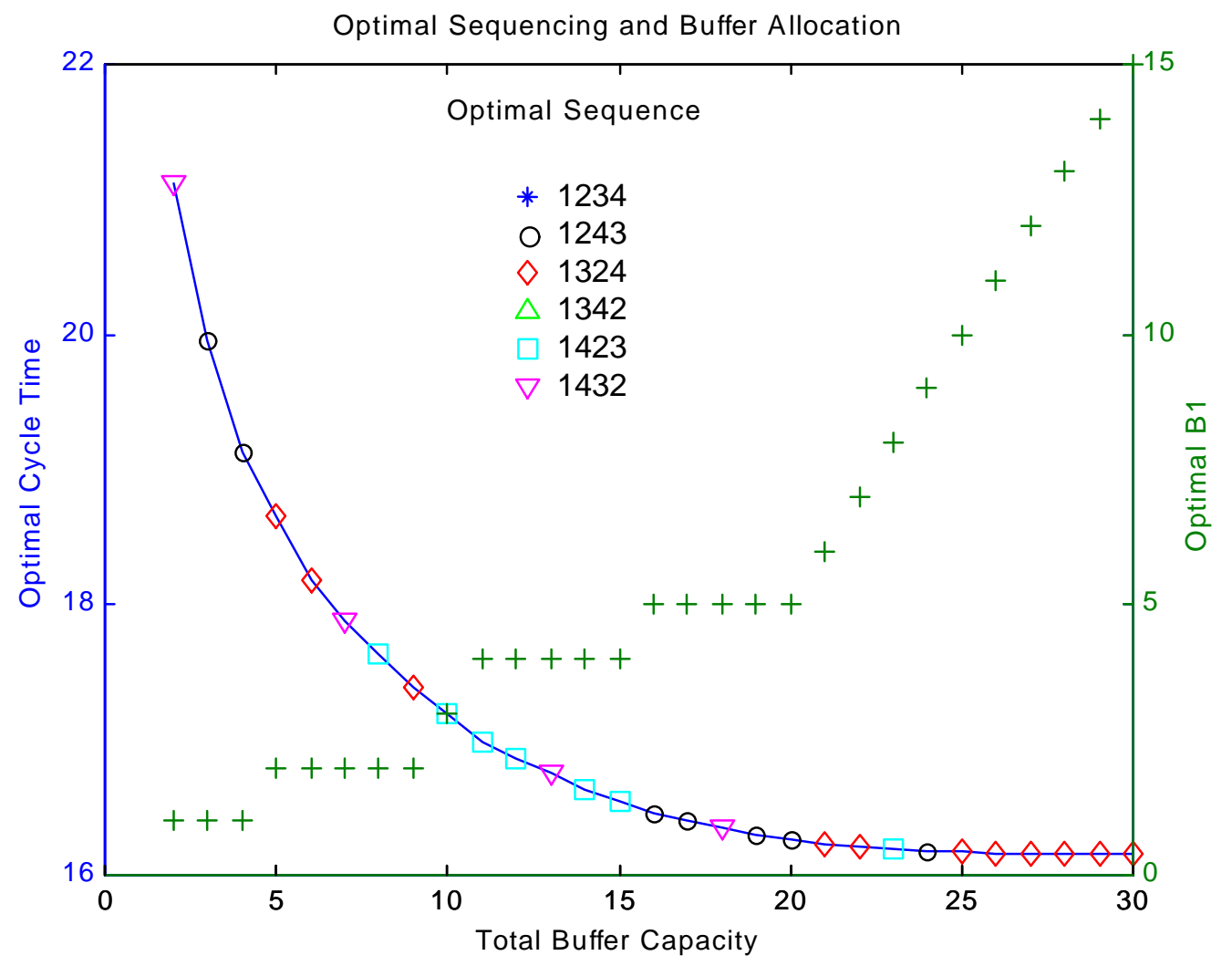

Figure 7 The optimal cycle time, the optimal sequence and the optimal buffer allocation that minimizes the cycle time.

In order to capture the effects of variability on the performance without increasing the computational requirements considerably, service times in a wide variety of production systems are modeled by using only two phases. ${ }^{10}$ Therefore, we use a balanced-mean two-phase Coxian distribution ${ }^{12}\left(C_{2: \mathrm{b}}\right)$ in the following numerical experiments. In a balanced-mean two-phase Coxian distribution, a job receives an exponential service first, and then either it finishes its operation on that machine and moves to the next one, or it stays on the same machine for another exponential service. This distribution can be used to approximate service time distributions that have a squared coefficient of variation of $1 / 2 \mathrm{r}$ higher. The reader is referred to Johnson and Taaffe ${ }^{19}$ for fitting distributions of phase type.

The processing rate, $\mu_{i, j}$, and the probability of receiving the second service, $q_{i, j}$, completely determine $C_{2: b}$. The squared coefficient of variation of the service time determines the probability of receiving the second service: $q_{i, j}=\left(2 s c v_{i, j}\right)^{-1}$. In the numerical experiments, we set the squared coefficient of variations of all the processing times to the same $s c v$ value. Note that the methodology presented in this paper can be used for general phase-type distributions.

Figure 8 shows the effects of variability on the optimal sequence and also on the optimal cycle time for both asynchronous and synchronous part transfer cases. We consider a three-station line producing 4 parts cyclically with no interstation buffers. Processing time of each job on each machine is $C_{2: \mathrm{b}}$ and their means are given in $4 \times 3$ matrix $[[8,5,6] ;[4,7,5] ;[9,2,3] ;[5,6,9]]$. The results for the synchronous cyclic production line are obtained by using the results presented in Karabati and Tan. ${ }^{2}$ Karabati and Tan present exact results for synchronous production lines with Weibull processing times. Since we consider phase-type distributions in this 
study, the synchronous results are obtained by representing the phase-type distribution with a corresponding Weibull distribution with the same three moments. As expected, the asynchronous line yields lower cycle time. The optimal sequence for this specific asynchronous line stays the same as the variability increases and it switches to another sequence for the synchronous case. In general, the variability alters the optimal sequence that minimizes the cycle time.

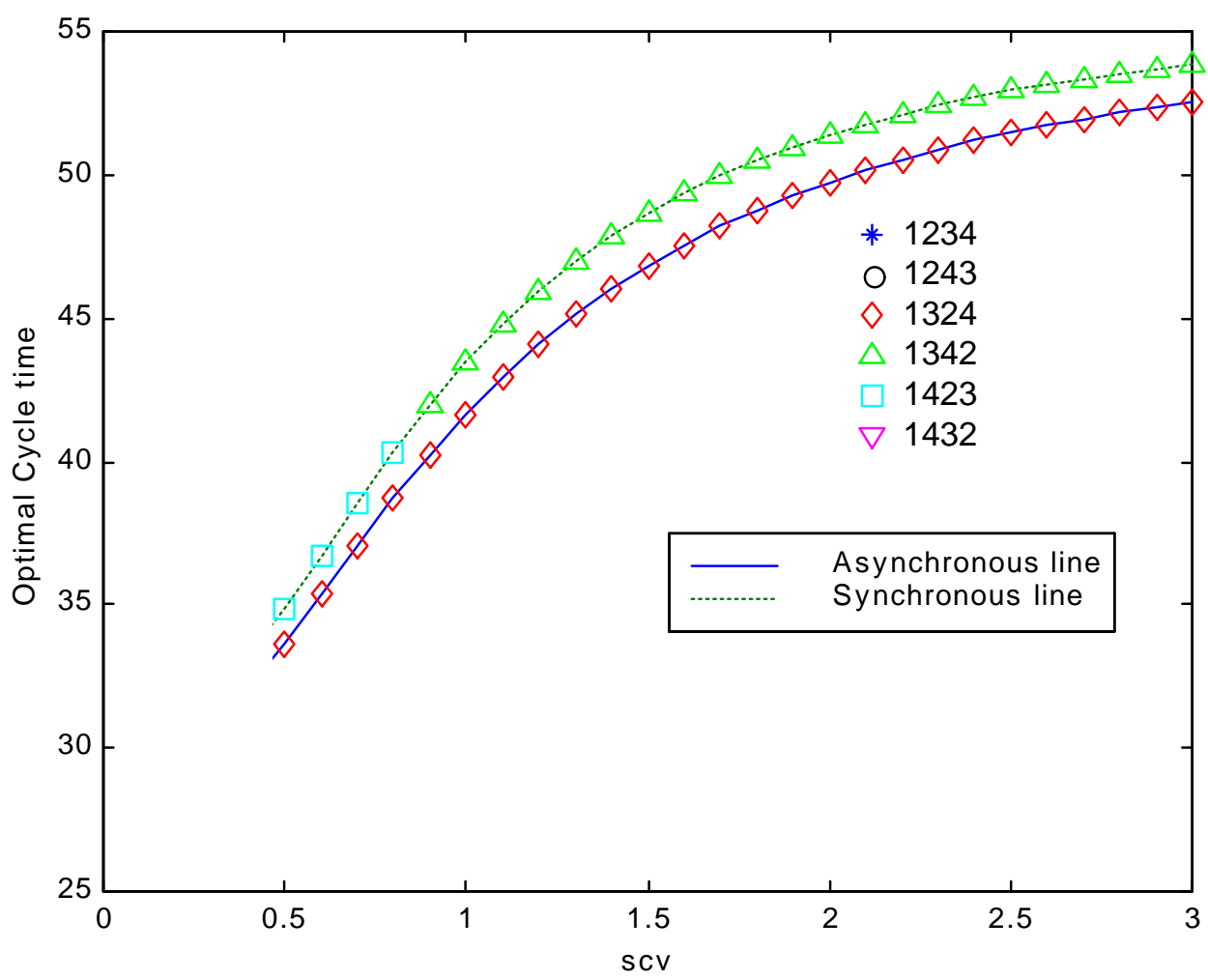

Figure 8 The effect of variability on the optimal cycle time and on the optimal schedule.

The throughput rate of the sequence that minimizes the deterministic case can be used as an approximation. Figure 9 depicts the percentage gain that will be obtained by using the optimal sequence compared to the optimal solution of the deterministic case for a specific three station production line with four jobs and no interstation buffers. Processing time of each job on each machine is $C_{2: b}$ and their means are given in $4 \times 3$ matrix $\quad[[0.3932, \quad 0.4586, \quad 0.1603] ; \quad[0.5915,0.8699,0.8729] ; \quad[0.1197,0.9342,0.2379]$; $[0.0381,0.2644,0.6458]]$. The figure shows that the optimal solution of the deterministic case provides a reasonable approximation to the stochastic sequencing problem. The maximum gain is $4 \%$ for the synchronous part transfer case and $3.3 \%$ for the asynchronous part transfer case. Since the percentage gain is $0 \%$ for $s c v=0$, the percentage gain should be first increasing and then decreasing as the processing time variability increases. When the $s c v$ approaches zero, the gain is small because the deterministic processing time approximates the processing time distribution well. When the scv increases, the scheduling problem becomes less relevant, because all the sequences yield similarly worse results.

Finally, Figure 10 shows the effect of variability and also the effect of the part transfer mechanism (synchronous vs. asynchronous) on the cycle time, on the WIP inventory and on the batch WIP inventory of a production line with three stations, four jobs, and no interstation buffers. Processing time of each job on each machine is $C_{2: \mathrm{b}}$ and their means are given in $4 \times 3$ matrix [[ $\left.0.8420,0.1304,0.4143\right],[0.1598,0.0910,0.0269]$, 
$[0.2128,0.2746,0.7098],[0.7147,0.0030,0.9379]]$. As the figure shows, asynchronous part transfer yields a shorter cycle time. Furthermore, the figure shows that reducing variability shortens the cycle time for both cases. When the expected number of parts is considered, asynchronous part transfer yields much better performance compared to the synchronous part transfer case. However, when the number of batches are considered, the performances of both cases are comparable.

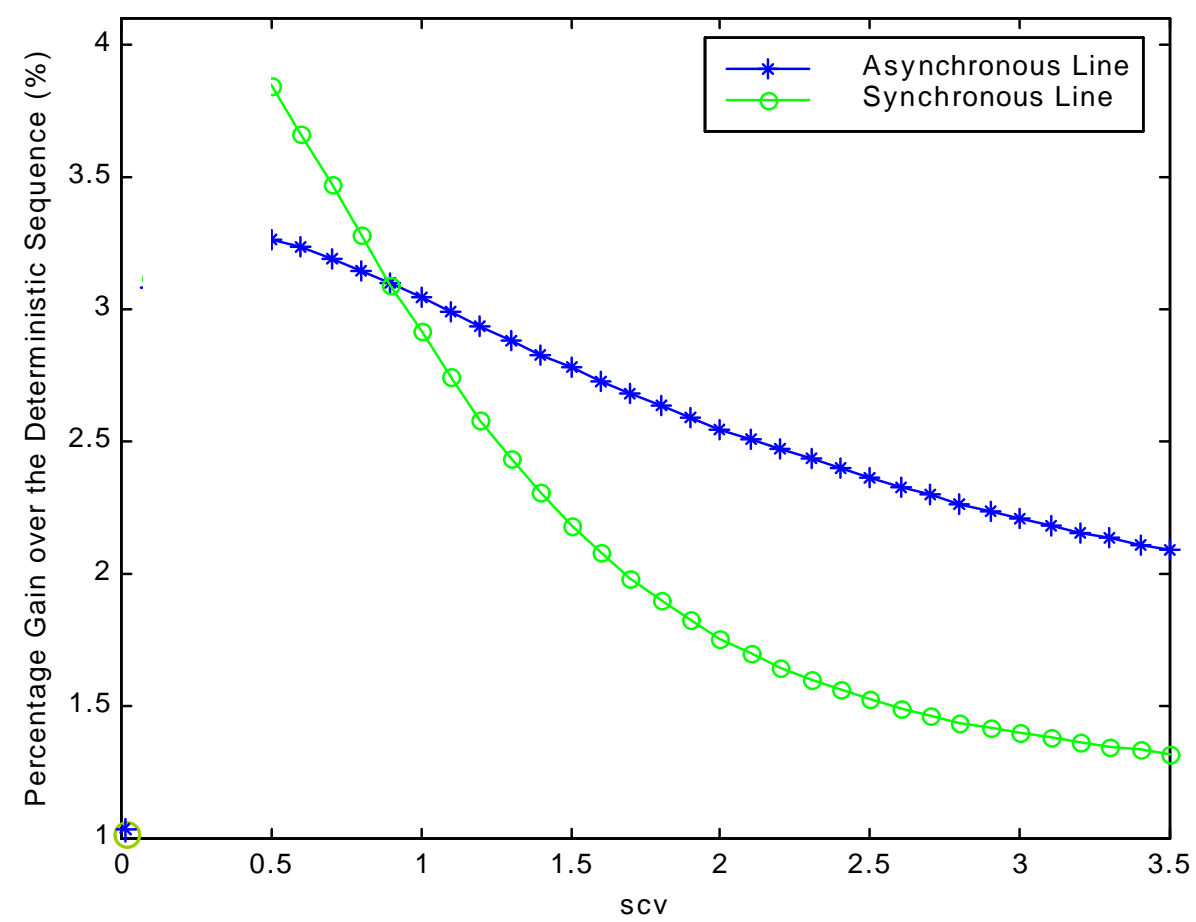

Figure 9 The effect of variability on the percentage gain obtained.
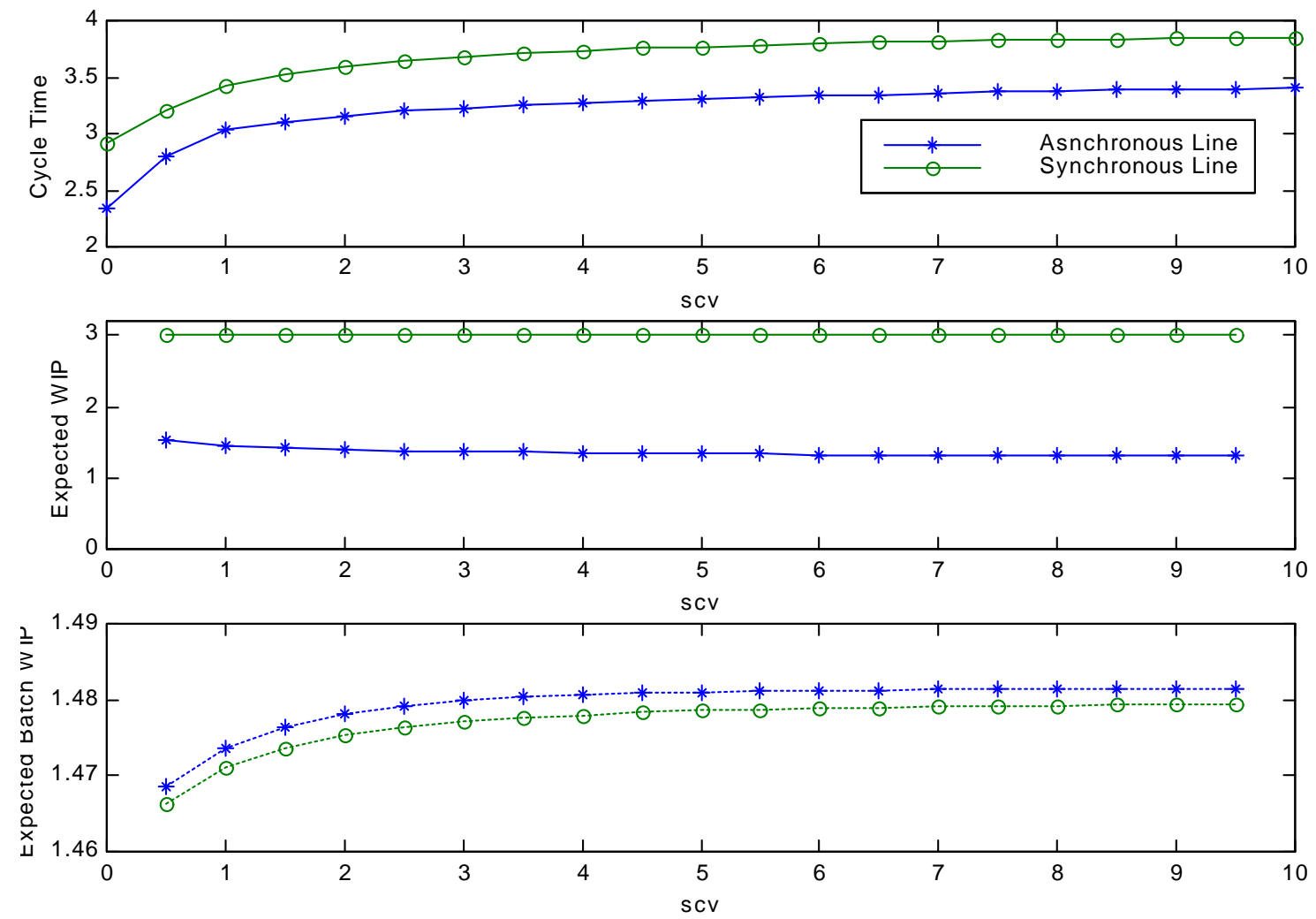

Figure 10 The effect of variability and the part transfer mechanism on the cycle time, on the WIP inventory and on the batch WIP inventory. 
Note that these observations are quite intuitive and some of them are proven for general discrete event systems. However, in order to design and operate production systems more efficiently, these observations must be quantified. The performance evaluation component of this methodology provides these numbers. For example, it is proven that asynchronous part transfer yields shorter cycle time (and higher production rate) compared to synchronous part transfer. This result, however, does not state how much better it performs, or whether this advantage continues in the same way when variability increases or decreases. The performance evaluation methodology presented here answers these questions objectively.

\section{Conclusion}

In this study, we model and analyze a production line with asynchronous part transfer, processing time variability, buffer allocation, and cyclic scheduling in the same framework. This approach allows one to investigate the interactions among processing time variability, operating decisions, production line structure and its performance.

When the cycle time (or production rate) is used as the primary performance measure, the performance of the asynchronous part transfer case is better than the performance of the synchronous part transfer case. Variability reduction has been subject to numerous quality programs. As the variability of the processing time increases, the performance of the line gets worse for both cases, or equivalently, as the variability decreases, the performance of the line gets better. Therefore, in order to improve the performance of a synchronous production line, one alternative is to change the way parts are transferred from synchronous to asynchronous part transfer. This requires heavy investment, since the structure of the production plant needs to be changed. However another simple alternative is reducing the variability. Under certain conditions, it might be possible to realize a comparable improvement in the performance of a synchronous line by reducing variability compared to switching to asynchronous part transfer. Similarly, the performance of the system can be improved by placing interstation buffers to absorb the effects of variability. In this case, although the production rate increases, WIP and as a result the time each part spends in the system also increases. It is important to note the effect of interstation buffer capacities. Reducing the interstation buffer capacities without reducing the variability may decrease the production rate of the system. In this case, it is important to determine the best allocation of the total buffer capacity.

Note that the model described in the first part of this study is not generative, i.e., it does not generate an optimal solution, rather it is an evaluative model that evaluates the performance of the production line for a given sequence. The output of this model has been used in a generative model presented in the second part to determine a near-optimal sequence for the production rate maximization problem.

The method for the asynchronous case uses the state-space representation of the model. Thus it suffers from exponential growth of the states as the number of stations and jobs increases. Performance evaluation of synchronous production lines are much easier than the determination of the asynchronous lines. Furthermore, the production rate of a production line with synchronous part transfers is a lower bound for the production rate of the same line with asynchronous part transfer. This property can be exploited. The effects of synchronous versus asynchronous part transfer on the performance of a production line and also on the operating decisions require further investigation. 


\section{References}

1 Schonberger RJ (1986). World Class Manufacturing. The Free Press, New York.

2 Karabati S and Tan B (1998). Stochastic cyclic scheduling problem in synchronous assembly and production lines. J Opl Res Soc 49: 1173-1187.

3 Magott J (1987). Performance evaluation of systems of cyclic sequential processes with mutual exclusion using petri nets. Inform Process Lett 21: 229-232.

4 Desrochers AA (1994). Applications of Petri Nets in Manufacturing Systems: Modeling, Control, and Performance Analysis. IEEE press: Piscataway, N.J.

5 Campos J, Colom J and Silva M (1990). Performance evaluation of repetitive automated manufacturing systems. In: Proceedings of Rennselaer's Second International Conference on Computer Integrated Manufacturing (Troy, NY). IEEE Computer Society Press, pp 74-81.

6 Rao US and Jackson PL (1996). Estimating performance measures in stochastic cyclic schedules. IIE Trans 8: 229-939.

$7 \quad$ Bowman R and Muckstadt J (1993). Stochastic analysis of cyclic schedules. Opns Res 41: 947-958.

8 Lee TE and Seo JW (1998). Stochastic cyclic flow lines: non-blocking, Markovian models. J Opnl Res 49: $537-548$

9 Zhang H and Graves S (1997). Cyclic scheduling in a stochastic environment. Opns Res 45: 894-903.

10 Dallery Y and Gershwin SB (1992). Manufacturing flow line systems: A review of models and analytical results. Queueing Syst 12: 3-94.

11 Buzacott JA and Shantikumar JG (1993). Stochastic Models of Manufacturing Systems. Prentice Hall, Englewood Cliffs, New Jersey.

12 Yeralan S and Muth EJ (1987). A general model of a production line with intermediate buffer and station breakdown. IIE Trans 19: 130-139.

13 Papadopoulos HT (1995). The throughput rate of multistation reliable production lines with no intermediate buffers," Opns Res 43: 712-715.

14 Neuts MF (1981). Matrix-geometric Solutions in Stochastic Models: An Algorithmic Approach. John Hopkins Press, Baltimore.

15 McCormick ST, and Pinedo ML and Wolf B (1987). The complexity of scheduling a flexible assembly system. Working paper. Management Sciences Department, School of Business, University of British Columbia, Vancouver BC, Canada.

16 Tan B and Karabati S (1997). Effects of variability on the performance of a production line under cyclic scheduling. In: Kaylan AR and Lehmann A (eds). Proceedings of the 1997 European Simulation Multiconference, pp 795-802.

17 Stewart WJ (1994). Introduction to the Numerical Solution of Markov Chains. Princeton University Press, New Jersey.

18 Papadopoulos HT and O'Kelly MEJ (1989). A recursive algorithm for generating the transition matrix of multistation series production lines. Comp Ind 12: 227-240.

19 Johnson MA and Taaffe MR (1991). An investigation of phase-distribution moment-matching algorithms for use in queueing models. Queueing Syst 8: 129-148.

20 Matsuo H (1990). Cyclic sequencing problems in the two-machine permutation flowshop: Complexity, worst-case and average-case analysis. Nav Res Logist. 37:679-694.

21 Gilmore PC and Gomory GE (1964). Sequencing a one state-variable machine: a solvable case of the traveling salesman problem. Opns Res 12: 655-679. 